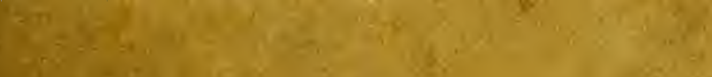

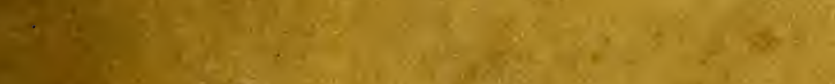

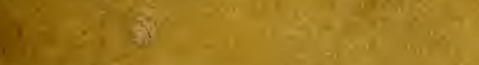

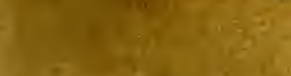

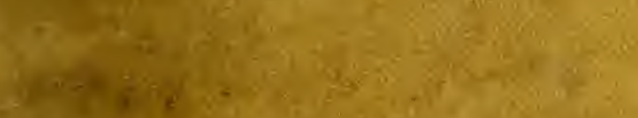

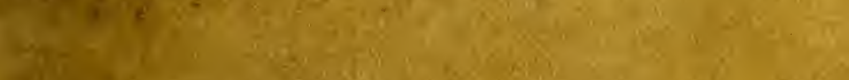

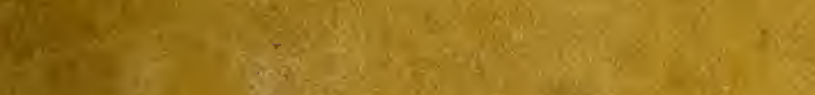

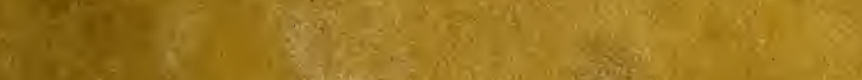

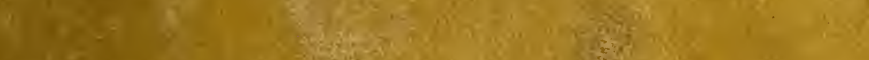

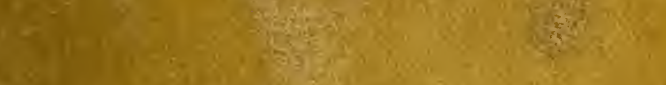

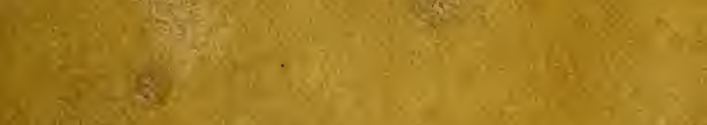

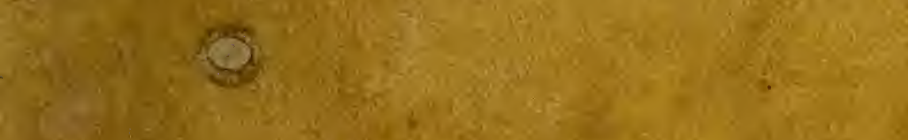

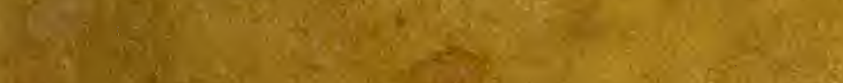

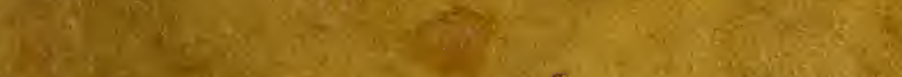

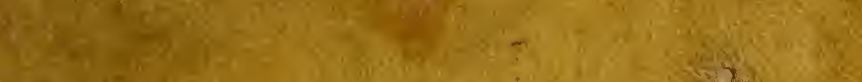

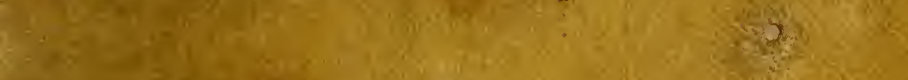

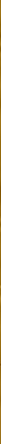




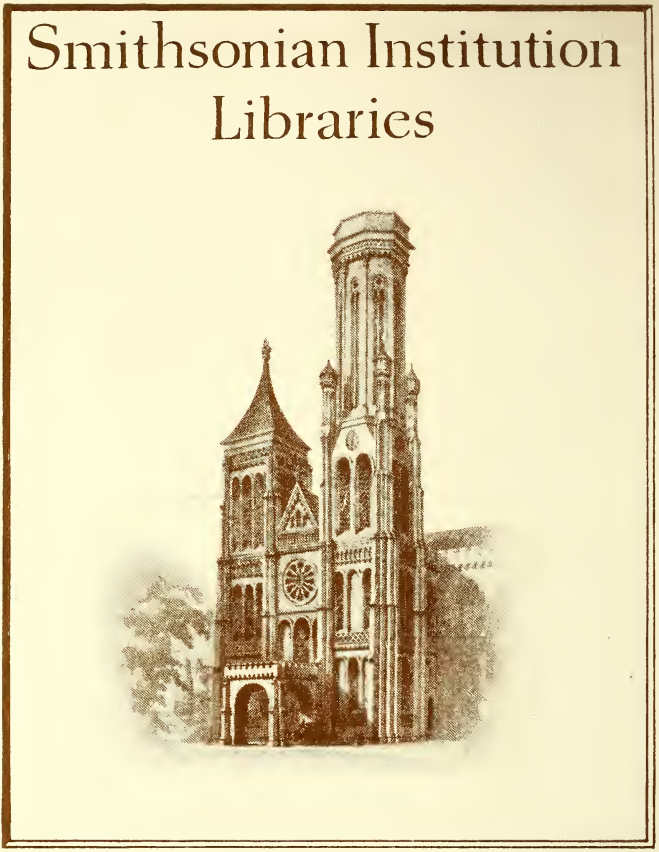





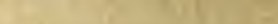

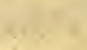

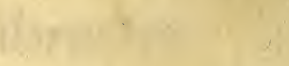

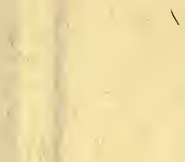

1
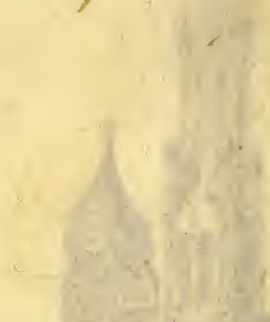

$(6+1,+5)$

$x^{2}+1$,

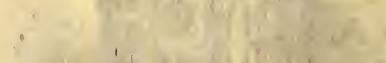

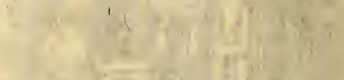

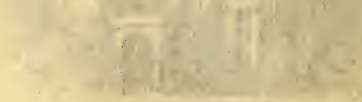

1

ins 


\section{B E A U}

\section{CABINET D'HISTOIRE \\ NA TURELLE}

A VENDRE A FLORENCE.

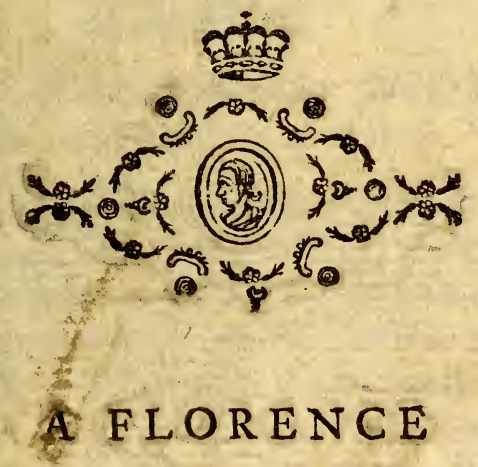

Chez $\mathrm{P}$ Allegrint à la Croix rouge. Avec approvation.

MDCCLXXXII. 


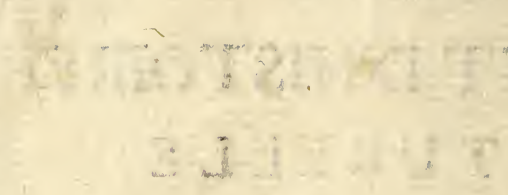

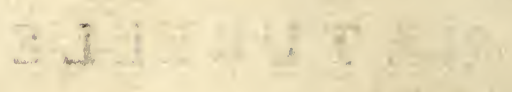

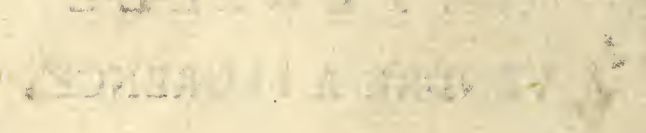

$$
\text { isitum }
$$

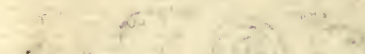

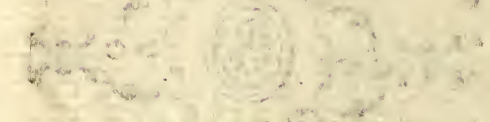

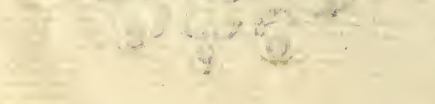

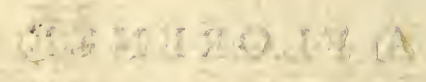

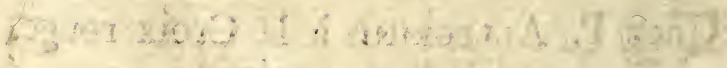

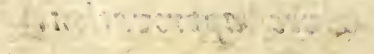

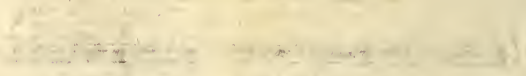

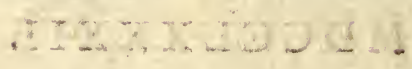


M58

$R B$

\section{B E A U}

\section{CABINET D'HISTOIRE \\ NATURELLE \\ A VENDRE A FLORENCE.

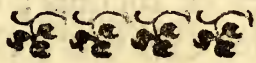

Une fuitte de Coquilles de Mer, felon l'ordre et la clafjation des Autbeurs.

1. $\left\{\gamma_{0} \mathrm{~N}\right.$ tiroir comprenant les lepas, Q3 ou Pâtelles des Mers diverfes, favoir de la Méditerranée, de l'Océan, $\&$ de l'Amerique, toutes différentes, grandes \& petites, on y voit celles du Magellant, il y a auffi des Cabochons, $\&$ fur tout le petit de la Chine, $\&$ des Dentales divefes .

2. Un tiroir comprenant des Nautilles des diverfes Mers, il y a entr'autre un beau Cimbium, ou la grande Nautille papirafée, \& une moins grande, il y a auffi 2 belles autres Nautilles de l'Océan, dont 


\section{SUITTE DES COQUILLES}

une gravée. Plus des creilles de plufieurs grandeures \& grofleures, \& une fort grande. Plus plufieurs Nerites à bouche ronde \& femi-ronde.

3. Un tiroir comprenant les Cochlées ou Efcargots, on y voit ceux de la Chine; il y a des beaux trochus de diverfes groffeures \& formes; on y voit les Dauphins, les Cadrans. Cette fuitte eft tres-belle.

4. Un tiroir comprenant les Buccins de diverfes groffeures \& couleures, on y voit les fufeaux. Plus fuivent les Viffes, ou les Turbinées, \& les Strombes, on y remarque la Thiare \& la Mitre.

5. Un tiroir comprenant les Cylindres, ou Rouleaux de diverfes efpeffes \& groffeurs, comme auffi les Pavées, les Pictées, \& autres éfpéces, comme les Olives .

6. Un tiroir comprenant les Murex, les Pyriformes, têtes de Becace épineufes, \& autres en bonne quantité.

7. Un autre tiroir comprenant auffi. des Murex, ou Rochérs des Mers diver- 


\section{SUITTE DES COQUILLES}

3

fes. Cette claffe eft nombreufe .

8. Un tiroir contenant les Tonnes, \& Calfidiformes de plufieurs grandeures \& formes.

9. Un tiroir comprenant les Porcelaines de beaucoup d' éf peces, on y voit le Belargus, il y a celles d'Amerique, les Oeufs, les Barcées, les Draps d' or, $\&$ autres.

io. Un tiroir comprenant les Huitres diverfes, \& Huitres épineufes toutes différentes entr'elles.

ì. Un tiroir comprenant les Cammes, on y en voit de diverfes Mers, comme de l'Océan, de l'Amerique, de la Méditerranée, \& du Magellan.

12. Un tiroir comprenant les pinnes, $\&$ les Moules, celles d'Amerique, ou du Magellant, \& de la Mediterranèe. Claffe nombreufe ,

13. Un tiroir comprenant les Coeurs dit Coeurs de boeuf, ou Bucarde, les Coeurs de Venus diverfes, les Huitres tuillés, \& les Crêtes de coq.

14. Un tiroir comprenant les péctiA 2 


\section{SUITTE DES COQUILLES}

nites, ou Cappes de Saint Jacque de diverfe groffeures \& grandeures variées en couleur.

I 5. Un tiroir de Muttivalves, comme les Vermiculaires, les Glands, les Pholades, les Natiferes, les Pouffes-pieds d'Amerique.

16. Un tiroir comprenant les Echinites, ou Heriffons de Mer, on y voit les éfpeces rares, comme les Mamilloné, ou Tuberculeux, les Spatagus differents, \& le Gateaux.

17. Un tiroir contenant diverfes éfpeces de Cruftaces, comme des Crabes, des Efquilles, des Cancres. Toutes de la Mediterranée.

Plus un tiroir comprenant diverfes éfpeces de Coquilles fluviatilles. 


\section{SUITTE DES FOSSILES}

Claffe des Petrifications et des Foffles tant Plantes que Coquilles.

18. भ $\gamma \mathrm{N}$ tiroir comprenant des Plantes marines petrifiées que les Naturelliftes appellent Plantes diluviales comme les Corails Coralloides, les Madrepores, les Aftroires, les Fungites. Cett eclaffe eft confiderable.

19. Un autre tiroir comprenant à-peuprès les mêmes éfpeces connuês auffi fous le nom fte Polypiers.

20. Un tiroir comprenant les diverfes éfpeces d'Huitres folfiles de diverfe figure \& éfpece, telles que'Bourguet \& Langius les ont publiées.

21. Un tiroir comprenant la claffe des Gryphites, \& Camites des différentes parties de l'Europe, favoir de France, de Champagne, d'Allemagne, \& d'Italie.

22. Un tiroir comprenant auffi des Cammes, des pines, \& des Moules de diverfes parties de l' Europe.

$$
\text { A } 3
$$




\section{SUITTE DES FOSSILES}

23. Un tiroir comprenant les Pectinites tres-belles, bien confervaes, on y voit la fol fofile rare

24. Un tiroir comprenant les Cochleites, les Trochites, les Tonnes, les Harpes de differentes parties de l'Europe, comme de l'Italie, de l'Allemagne, \& de la France.

25. Un tiroir comprenant des Murex de differentes parties de l'Europe, pieces tres-intcreffantes ammaffées eu Italie, en Allemagne, \& en Francc.

26. Un tiroir comprenant les Turbinés, ou les Viffes des diverfes parties, comme du Veronois, de la Champagne, $\&$ de la France.

27. Uu tiroir comprenaat les Noix de mer, les Figues \& Porcellaines ammaffée en Champagne, en Italie, \& dans les Faluns de France.

28. Beau tiroir comprenant une fuitte d'Echinites, ou Heriffons de diverfes parties de l'Europe, de l'Italie, de la France, de la Bourgogne, \& de l'Angleterre convertis en Silex. Suitte curieufe: 
29. Un autre tiroir comprenant diverfes efpeces auffi d' Echinites de figure \& forme aulfi diverfes, de differents pays.

30. Un tiroir contenant des Vermiculaires de qualité \& d' efpece, qui ne font pas bien connus, ni decrits par les Naturaliftes, ammaffés en differentes parties de l'Europe.

3 I. Un grand tiroir comprenants environ 100. boếtes de diverfes grandeurs, ou font des corps marins relatifs aux animaux, ou aux plantes, ou Coquilles, objets tres-intereffants pour l'Hiftoire naturelle ammaffées en Italie, en Allemagne, \& en France.

32. Un grand tiroir rempli de cornes d'Ammon diverfes en grandeur \& forme, ammaftées en diverfes parties de l'Europe, objet tres-beau pour la grandeur, \& les efpeces.

33. Un autre tiroir rempli d' objets à-peu-près femblable bien confetvés, curieux, \& variés.

34. Un grand tiroir comprenant des $\mathrm{A}_{4}$ 


\section{- SUITTE DES FOSSILES}

grands objets de perrification d' un vom lume, ou grandeur remarquable, comme Huitres, Cammes, Pectinites, \&c.

35. Un autre grand tiroir comprenant environ 100. boetes, ou vafes rempli de Corps marins, qui fignifient beaucoup pour l' hiftoire naturelle

36. Un grand tiroir comprenant environ 40. Impreffions ou Squelletres de Poilfons petrifiés, le tout bien exprimé \& remarquable. Plus des Oolites ou oeufs de poiffons ammaffées en diverfes parties de l' Europe, comme en Italie, France, \& Allemagne.

37. Autre grand tiroir comprenant des empreintes des Plantes terreftre \& marincs, Feuilles de fougeres, \& corallines \&c.

38. Un autre tiroir comprenant des Os d'animaux diverfes, foit foffiles, ou autres, comme des cornes d'Elephant des Os des Vertebres, des Jambes, Dents, Machoires, \& autres pieces . 39. Autre caffette des mêmes efpeces. 


\section{Clafje de Mineralogie.}

40. If $\mathrm{N}$ tiroir comprenant des $\mathrm{Mi}$ nes d' or , \& d' argent, \& de plomb, fcavoir dix à donze murceaux de Mine, d' or bien evident \& claire des differentes parties du monde, favoir du Perou, de la Hongrie, d' Allemagne, \& de France. Plus des morceaux de Mine d'argent de Pano, au Perou, de Saxe, de Hongrie, de France \&c.

4I. Un autre tiroir comprenant des Mines a' argent \& de plomb de diverfes richeffes \& couleurs, d'argent gris, de plomb vert, du plomb gris, contenant aufi de l'argent au nombre de 36 . morceaux .

42. Un' autre caflette des Mines de Cuivres de differentes partics d'Europe, de la France, de la Savoye, de I'Italie, de l' Alemagne, \& autres, au nombre de 3 I. morceaux. 


\section{so SUITTE DU CABINET}

43. Un' autre caffette comprenant des Mines de Cuivre de France, d' Italie, d'Allemagne, \& d' Hongrie, au nombre de 30 . pieces.

44. Une caffette comprenant des Mines de fer de diverfes figures \& richeffes, am maffées en diverfes parties d'Europe, favoir en Italie, en France, \& en Allemagne, au nombre de quarante efpeces.

45. Une caffette contenant diverfes Mines de Mercure, \& de Cinabre provenant de l'Efpagne, de l'Hiftrie, de l'Italie, de ia Hongrie, \& des Indes, aux nombre de 24. efpeces.

46. Un tiroir contenant des Pyrites des Marcaffite, des Feluors fous diverformes, de differentes parties de l'Europe.

47. Un tiroir qui contient encore des Marcaffites, des Feluors, \& des Semi-metaux, comme de l'Antimoine, de l' Etain \&c. au nombre de vingt-quatre fortes.

49. Un autre tiroir contenat des Sou- 
fres, des Matieres Semi-metaliques, Arfenicales, \& auffi diverfes autres pieces, au nombre de quarante-deux qualités.

49. Plus un autre tiroir contenant des Sels des Souffres, des Vitriols, d'Alum, de plufieurs endroits de l'Europe, \& notammert de l'Italie, de l'Allemagne, du Tirol \&c. au nombre de vingt-quatre fortes .

50. Il y a pareillement un tiroir comprenant des Conglumerations metalliques, comme des Geodes, des Etites, des Manganeifes \&c, au nombre de 24. pieces.

51. Uir tiroir comprenant des Bitumes des 'Tourbes, des Charbons foffiles, ou autrement appellé Charbon de pierre, de plufieurs endroits de l'Europe, favoir de la Flandre, de l'Italie, de la France, de l'Angleterre, \& dè l'Allemagne, au nombre de vingtquatre efpeces.

52. Un' autre tiroir comprenant les produits Vulcaniques, c'eft-à-dire les 
laves, les Piperites, les Scherles, ou Schroles Pierres fondues de divers Volcans , favoir de l' Italie, de la Sicile, du Veronois, de la Tofcanne, \& autres endroits, au nombre de 24 . fortes .

53. Un autre tiroir contenant auffi des produits Vulcaniques, favoir de la Lave, des Bafaltes, Pierre ponfe, Pierre fondue, connue fous le nom de Granite du Piperites, le tout au nombre de 25. efpeces, foit de l'Italie, ou de l'Allemagne, \& d'autres endroits .

Plus un grand morceau de colonne de Bafaltre des Monts Eganeens qui font dans le Frioul. 


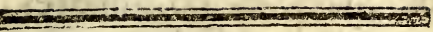

\section{SUITTE D' HISTOIRE NATURELLE}

Comprenant les Pierres Calcaires, et autres Pierres propres à bâtir, on à faire de la Cbalix et à tailler pour l'Architecture.

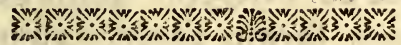 \\ C A I A LOGUE}

Des Pierres, Marbres Albâtve, Cailloux, Granites, Iafpe, Brêche, Agatbe, pierres dures.

I. RRemierement une caffette conlie tenant 30. qualités d' Albâtre de differentes parties d' Europe. 2. Une caffette, ou petit tiroir comprenant des carrés de differentes Pierres \& Cailloux, pris dans l'Arno, foit 


\section{SUITTE DES MARBRES}

en Pierre, ou matieres coagulaires, en des endroits prifes dans l' Arno, \& dans les environs de Florence, au nombre de 28. efpeces.

3. Autre caffette contenant trent-huit efpeces de Marbres differents, taillés en carrés eguaux entre eux, pris en diverfes parties de l'Europe, \& particulicrement de 1'Italie, de la France, de l'Allemagne \&c.

4. Une caflette contenant des Brêches de mêmes forme carrées au nombre de 19. fortes. Plus des pieces de Granite de même forme, pris en diverfes parties de l'Europe, \& de l'Afie, foit en Egypte, foit en Italie dans les Monts du Milanois, \& du Tirol, de la Tofcanne \&c, au nombre de vingt morceaux ou pieces egales.

5. Une autre petite caffette, ou tiroir comprenant trente-cinq formes carrées de Marbre de differentes qualités prorenant des divers endroits, favoir de la Sicile, du Milanois, de la Tofcanne, de l'Afrique, \& d'autres parties. 


\section{SUITTE DES MARBRES}

15

6. Un tiroir comprenant vingt-neuf autres formes egalles de Marbre, \& de Brêche de differantes efpeces

7. Un tiroir rempli des Pierres dites Serpentines Gabre, Pierre Ollaire, ou Steatites, au nombre de trente-cinq qualités.

8. Un tiroir comprenant des carrés de pierres dites Laves piperin, de divers Volcans de I'Italie, au nombre de trente-cinq qualités. Plus dix-huit autres echantillons de Laves diverfes.

9. Un tiroir comprenant des carrés de Pierre organifées, commeLumachelle, Pierre etoilées, ou aftroites Cerebrite, \& pierres de cette nature, au nombre de trente-trois .

10. Un tiroir rempli d' environ 100. Pieces, foit Agathe, Coraline, Jafpe, \& pierres fines fous des formes oval les, recueillies en diverfes parties du Globe.

I I. Plus 50. autres pieces de Pierre à-peu-près de lạ même nature fous des formes plattes \& arrondies. 


\section{-16 SUITTE DES MARBRES.}

I2. une Caffette comprenant cinquante formes, ou carrés des Pierres fines, en Jafpe rares, comme Jafpe ranguin, Agathe, Jafpe de Boheme, de Sicile. du Levant, \& autres objets intereflants.

14. Une caffette remplie d'autres Jarpes en forme carré, \& oval, \& ronde, toute en Jafpe, Agathe \& Pierres fines. Objets intereflant $\&$ rares.
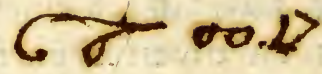


\section{$S U I T T E$}

D'HISTOIRE NATURELLE

\section{COMPRENANT}

Les Tartarifations, les Incruftations, les Pierres Brutte, et figurée. Les Sables, les Cailloux etc. Et pierres comunes, et les Crifteaux, et quartz.

54. $\Upsilon$ Y $\mathrm{N}^{\prime}$ autre caffette comprenant encore les produits Vulcanique de la Tofcanne, du Vefuve, de la Sicile, de la Gorgone, de l'Etat de Rome, des Bafaltes de la Gorgone au nombre de 25 . fortes.

55. Un tiroir contenant des Tartarifations, \& des Incruftations que produifent differentes eaux naturelles, \& minerales des diverfes parties de l' Europe, \& fur tout de la Tofcanne au nombre de 32. efpeces. Plus des StaB 


\section{SUITTE DU CABINET}

lagmites, \& Stalactites differemmene figurée.

56. Un autre tiroir comprenant aufli des Tartarifations. Plus des Selenit des Gipfes des diverfes parties de la Tofcanne.

57. Un tiroir comprenant des Spates de plufieurs efpeces, \& de plufieurs pays differemment configurée, au nombre de trente qualités diverfes.

58: Un tiroir comprenant des Spates de diverfes autres efpeces que ci-deflus au nombre de trente qualité.

45. Un tiroir comprenant des Sables ammaffés dans des Mers d'Iralie \& du Nord, \& dans diverfes Fleuves, $\&$ Rivierés de l' Europe, au nombre de foixant-un : Ceux-ri pouroient faire voir la nature des Pierres \& Sables fur lefquelles coulent fes eaux, tant de la France, que de l'Allemagne, \& les 'Torrents de l'Italie.

60. Un tiroir comprenant des Crifleaux, des Criftallifations, \& des quariz des diverfes parties; foit Monts 


\section{SUITTE DU CABINET}

ou Cavernes de l' Europe, comme de l'Italie, de la Hongrie, de la Bóleme, de l'Allemagne, \& de la Savoye au nombre de 42 . efpeces.

6r. Un tiroir comprenant des Cailloux ou Silex tant en Pierres dures \& fines, comme Agathe, Jafpe, Granite, Mierre figure calcaire, ammaffée en difiercntes parties des Monts, \& Fleuves d'Europe, \& du Levant, au nombre de 30. efpeces.

62. Un tiroir comprenant des Pierres dures, \& criftallifées, comme Agathe, Ametifte, Grenats \&c. au nombre de vingt-quatre efpeces .

63. Plus des Pierres ou Globes ditre Ventri Gemmati de diverfes figures, comme les Melons du Liban, \& autres.

64. Uu autre tiroir comprenant des Granites, \& Pierres de cette efpece qui prennent un poly brillant, ammaffées en diverfes Montagnes, \& parties d'Europe, \& du Levant, au nombre de 24. efpeces.

6.. Un tiroir comprenant des Talques 


\section{SUITTE DU CABINET.}

des Mica de diverfes parties de l'Europe au nombre de 24 . efpeces toute etiquetelées.

66. Un tiroir comprenant un fuitte d'Asbefte, \& d'Amiante, ammaffé en differentes parties du Globe, foit des Indes connue fous le nom de Lapis Cananor, ou de l'Europe fur le nom d'Amiante, comme de la Tofcanne, de Cypre, de Corfe, \& d' autres endroits, au nombre de 30 . fortes.

67. Un titoir comprenant des Gabres, des Serpentines, des Pierres favoneufe, dites auffi pierres ollaires, ou Cray d'Efpagne Smectis, ou Steatites, au nombre de 24. fortes.

68. Un tiroir où font les Pierres ditte Scifcilles, comme Ardoifes Cotis, Pierre de touche, \& pierre fableu fe divifible par lame, au nombte de vingt efpeces.

69 . Un tiroir comprenant des terres propres aux tintes, \& couleurs pour les arts, \& les Vafes fictiles, les ocre, les crayfes de diverfes parties de l'Europe. 


\section{SUITTE DU CABINET}

70. Un encognure comprenant diverfes Pifces, de Spate, de Stalagmit, des quartz, \& Stalactites, au nombre de environ 24. pieces.

71. Autre Encognure contenant des Criftallifations des quartz des Melon du Liban, \& autres pieces au nombre de vingt-cinq.

72. Un autre Encognure contenant des Spates, \& autres, au nombre de huit pieces. 


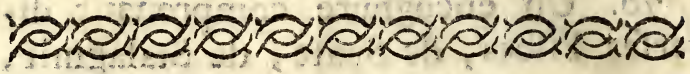

\section{GROSSES PIECES A ADJOUTER.}

1. a gr Lufieurs groffes Etites fous

5. d diverfes numcros.

6. \& 7. Plus deux dents d'Elephant du poids chaqu'un d'environ quatrevingt-dix livres.

8. Plus uue groffe'Tartarifation d'environ du poids de cent livres.

9. \& Io. Plus deux, ou trois gros morceaux de Ponfe, prife à l'imbouchure du Vulcan de Radicofani. Plus des groffes Coquilles pour mettre en evidence, toutes belles, au nombre de vingt.

Deux pieces de Roche de Mer pour mettre en couronnement fur les credences.

Plus douze groupes en forme de troffés fur lefquelles font adapté des belles Plantes marine des diverfes efpeces \& forme, qui font un bel effets mis en couronnement, avec d'autres. Plantes \& 
Coquilles, qui forment des Groupes agréables \& rares.

II. Plus deux grandes Credances, dont l'une comprend des Plantes marines, connuèsaufi fous le nom de Polipiers, comme les Coraux, les Coralloides, les Madrepores, les Acroporo, les Anguipores, les Pors cervins, les Cerebrites, les Fungites, les Dantelles, ou Retipors, les Coraux blancs, les Etoilée, ou Afroites, les Garophilites, Coraux noir, au nombre de 80. pieces contenuês dans des vafes, ou fur des bafes en piedeftal .

I 2. Un autre Credance, comprenant des Plantes marines, connuês fous le nom de Fucus d'Alcion, ou d'Eponges des Keratophites \& 2 . au nombre d'environ quatre-vingts, toutes dans des vafes de vetre, bien confervées .

Plus des Plantes marines enquadrées \& fous des verres pour les conferver, \& en jouir à la vuê, d'environ quinze Tableaux.

Plus deux grands morceaux d'Orguo 


\section{GROSSES PIECES}

¿'un volume rare, \& confiderable en couleur rouge.

Plus quatre Tableaux de poiffon deffechés fous des verres.

- 13. Une petite credence, où font arrangé des differentes mines de fer \& de marcafite, au nombre d'environ 40. pieces .

Plus une belle Corne de Rinoceros bien grande.

- Plus diverfes fruits des Indes.

Plus des Tableaux en pierre ditte d'endrites, ou pierre repprefentant.des ruines, ou des Villes incendiées, au nombre de dix.

14. Une belle fuitte de Bois Agatifé polits pour en montrer la nature, \& les efpeces, entre lefquelles des Joncs Agatifé, recuillies en diverfes parties de l'Europe. On y voit de grand pieces de Bois de Noyer, de Chêne, de Pins, \& de Sapins, tout objets rares, provenant des diverfes parties de l'Europe, comme de la Hongtie, de la Saxe, \& de l'Italie. Collection inte- 


\section{PIECES RARES.}

reffante, au nombre de foixante-une pieces groffes \& petites.

N. B. Un tres-beau Bezoard Oriental, gros comme un oeuf, du prix de cinquante Ducats .

Un Bezoard de Porc Epic Oriental du prix de vingt-cinq Ducats d' Holande.

Plus des petits Bezoards Orientaux, \& d' Amerique, \& diverfes pierres fines curieufes \& rares .

\section{NOTE.}

Il y a aufli chez le Pofleffeur de cet Cabinet une belle fuitte des Medailles antiques Romaines, au nombre de fept-cent-cinquante de grand bronze, qui commencent à Augufte jufque à Polthume.

Il y a auffi une fuitte des dittes Medailles Lattines, \& beaucoup de Grec relatives aux mêmés, en moyen bronze, toutes bien confervées depuis 
26 SUITTE DU CABINET . Augufte juffuu'à l'Empereur Poithume. Il y a encore une fuitte de fixcents Medailles Greques des Roi, des Villes, \& des Peuples, que l' oa vendra enfemble; ou par fuitte detachées.

Le Poffeffeur de touts ces objets eft Monfieur le Docteur B. Mesny Medecin de Cour de S. A. R. le GRAND DUC DE TOSCANNE \&c. A Florence. 

b) 



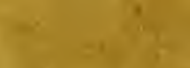

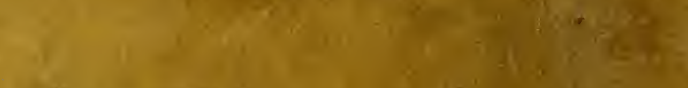

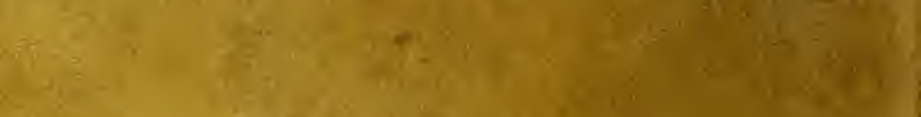

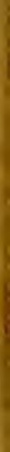

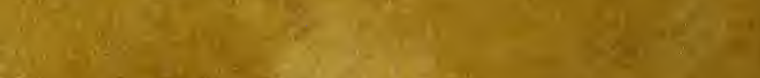

20.

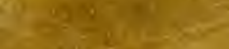

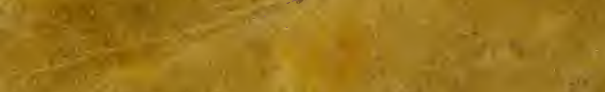

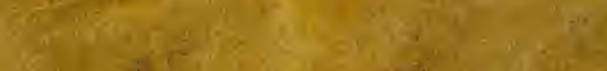

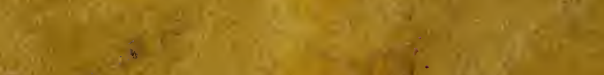

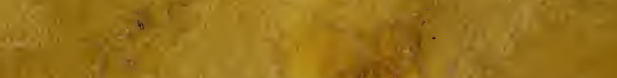

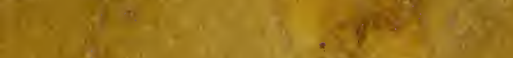

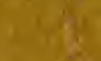

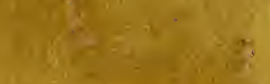

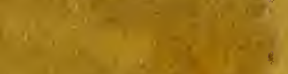

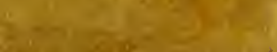

$66^{1}$

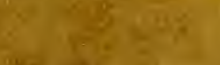

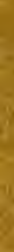

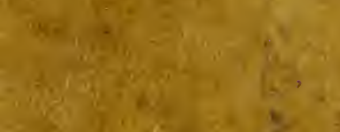
$x^{2}+x^{2}=3$

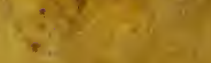

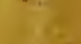

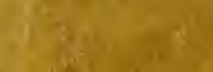
4 